\title{
Coincidence Point, Best Approximation, and Best Proximity Theorems for Condensing Set-Valued Maps in Hyperconvex Metric Spaces
}

\author{
A. Amini-Harandi, ${ }^{1}$ A. P. Farajzadeh, ${ }^{2}$ D. O'Regan, ${ }^{3}$ and R. P. Agarwal ${ }^{4}$ \\ ${ }^{1}$ Department of Mathematics, University of Shahrekord, Shahrekord 88186-34141, Iran \\ ${ }^{2}$ Department of Mathematics, Razi University, Kermanshah 67149, Iran \\ ${ }^{3}$ Department of Mathematics, National University of Ireland, Galway, Ireland \\ ${ }^{4}$ Department of Mathematical Sciences, Florida Institute of Technology, Melbourne, FL 32901, USA
}

Correspondence should be addressed to A. Amini-Harandi, aminih_a@yahoo.com

Received 8 October 2008; Accepted 9 December 2008

Recommended by William A. Kirk

In hyperconvex metric spaces, we first present a coincidence point theorem for condensing setvalued self-maps. Then we consider the best approximation problem and the best proximity problem for set-valued mappings that are condensing. As an application, we derive a coincidence point theorem for nonself-condensing set-valued maps.

Copyright (C) 2008 A. Amini-Harandi et al. This is an open access article distributed under the Creative Commons Attribution License, which permits unrestricted use, distribution, and reproduction in any medium, provided the original work is properly cited.

\section{Introduction and preliminaries}

The best approximation problem in a hyperconvex metric space consists of finding conditions for given set-valued mappings $F$ and $G$ and a set $X$ such that there is a point $x_{0} \in X$ satisfying $d\left(G\left(x_{0}\right), F\left(x_{0}\right)\right) \leq d\left(x, F\left(x_{0}\right)\right)$ for $x \in X$. When $G=I$, the identity mapping, and when the set $X$ is compact, best approximation theorems for mappings in hyperconvex metric spaces are given for the single-valued case in [1-4], for the set-valued case in $[1,3,5-9]$. Some results for condensing set-valued maps were given in [2].

Given subsets $A, B$, set-valued mappings $F: A \multimap B$, and $G: A \multimap A$ the best proximity problem consists of finding conditions on $F, G, A$, and $B$ implying that there is a point $x_{0} \in A$ such that $d\left(G\left(x_{0}\right), F\left(x_{0}\right)\right)=d(A, B)$. Then $\left(G\left(x_{0}\right), F\left(x_{0}\right)\right)$ is called a best proximity pair, see $[2,10]$. For $A, B$ nonempty subsets of a metric space $M$, we define the following sets

$$
\begin{aligned}
& A_{0}=\{x \in A: d(x, y)=d(A, B) \text { for some } y \in B\}, \\
& B_{0}=\{y \in B: d(x, y)=d(A, B) \text { for some } x \in A\} .
\end{aligned}
$$


A metric space $(M, d)$ is said to be a hyperconvex metric space [11] if for any collection of points $x_{\alpha}$ of $M$ and any collection $r_{\alpha}$ of nonnegative real numbers with $d\left(x_{\alpha}, x_{\beta}\right) \leq r_{\alpha}+r_{\beta}$, we have

$$
\bigcap_{\alpha} B\left(x_{\alpha}, r_{\alpha}\right) \neq \varnothing
$$

The admissible subsets of a hyperconvex metric space $M$ are sets of the form $\bigcap_{\alpha} B\left(x_{\alpha}, r_{\alpha}\right)$, that is, the family of all ball intersections in $M$. Every admissible subset of a hyperconvex metric space is hyperconvex. For a subset $A$ of $M, N_{\epsilon}(A)$ denotes the closed $\epsilon$-neighborhood of $A$, that is, $N_{\epsilon}(A)=\{x \in M: d(x, A) \leq \epsilon\}$, where $d(x, A)=\inf _{y \in A} d(x, y)$. If $A$ is admissible, then $N_{\epsilon}(A)$ is admissible [12].

A subset $A$ of a metric space $M$ is said to be externally hyperconvex if given any family $x_{\alpha}$ of points in $M$ and the family $r_{\alpha}$ of nonnegative real numbers satisfying

$$
d\left(x_{\alpha}, x_{\beta}\right) \leq r_{\alpha}+r_{\beta}, \quad d\left(x_{\alpha}, A\right) \leq r_{\alpha}
$$

it follows that

$$
\bigcap_{\alpha} B\left(x_{\alpha}, r_{\alpha}\right) \cap A \neq \varnothing
$$

Every externally hyperconvex subset of a metric space is hyperconvex [13, Theorem 3.10]. Let $(M, d)$ be a metric space and $X$ be a nonempty subset of $M$. $X$ is said to be a proximal nonexpansive retract of $M$ if there exists a nonexpansive retraction $r: M \rightarrow X$ with the property

$$
d(x, r(x))=d(x, X), \quad \text { for every } x \in X
$$

Every admissible set is externally hyperconvex and the externally hyperconvex sets are proximinal nonexpansive retracts of $M$ [14].

For each $A, B \subseteq M$, let

$$
d(A, B)=\inf \{d(a, b): a \in A, b \in B\} .
$$

It is well know that if $A$ and $B$ are compact subsets of $M$ then there exist $a_{0} \in A$ and $b_{0} \in B$ such that $d(A, B)=d\left(a_{0}, b_{0}\right)$. Therefore, in this case

$$
d(A, B)=0 \Longleftrightarrow A \cap B \neq \varnothing .
$$

Let $X$ and $Y$ be topological spaces with $A \subseteq X$ and $B \subseteq Y$. Let $F: X \multimap Y$ be a set-valued map with nonempty values. The image of $A$ under $F$ is the set $F(A)=\bigcup_{x \in A} F(x)$ and the inverse image of $B$ under $F$ is $F^{-}(B)=\{x \in X: F(x) \cap B \neq \varnothing\}$. Now $F$ is said to be

(i) lower semicontinuous if for each open set $B \subseteq Y, F^{-}(B)=\{x \in X: F(x) \cap B \neq \varnothing\}$ is open in $X$; 
(ii) upper semicontinuous if for each closed set $B \subseteq Y, F^{-}(B)=\{x \in X: F(x) \cap B \neq \varnothing\}$ is closed in $X$;

(iii) continuous if $F$ is both lower semicontinuous and upper semicontinuous.

Let $M$ be a metric space and let $\mathcal{M}$ denote the family of nonempty, closed bounded subsets of $M$. Let $A, B \in \mathcal{M}$. The Hausdorff metric $D$ on $\mathcal{M}$ is defined by

$$
D(A, B)=\inf \left\{\epsilon>0: A \subseteq N_{\epsilon}(B), B \subseteq N_{\epsilon}(A)\right\} .
$$

Let $X$ be a nonempty subset of $M$. A set-valued map $F: X \multimap \mathcal{M}$ is called Hausdorff continuous if it is continuous with respect to Hausdorff metric.

A topological space is said to be acyclic if all of the reduced Čech homology groups over rationals vanish. Every hyperconvex metric space is acyclic [15]. Let $X$ be an admissible subset of $M$. A set-valued map $F: X \multimap M$ is said to be quasiadmissible if the set $F^{-}(A)$ is closed acyclic for each admissible set $A$ of $M$.

Let $B(M)$ denote the set of all bounded subsets of $M$. The Kuratowski measure of noncompactness $\alpha: \mathbb{B}(M) \rightarrow[0, \infty)$ is defined by

$$
\alpha(A)=\inf \left\{\delta>0: A \subseteq \bigcup_{i=1}^{n} A_{i}, \operatorname{diam}\left(A_{i}\right)<\delta\right\}
$$

A mapping $F: M \rightarrow \mathcal{B}(M)$ is said to be condensing provided that $\alpha(F(A))<\alpha(A)$, for any $A \in \mathcal{B}(M)$ with $\alpha(A)>0$. If $\alpha(F(A)) \leq \alpha(A)$ for any $A \in \mathcal{B}(M)$, then $F$ is called 1-set contraction.

The following fixed point theorem, which will be used in the next section, is due to Amini-Harandi et al. [6].

Theorem 1.1. Let $M$ be a hyperconvex metric space. Suppose that $F: M \multimap M$ is an upper semicontinuous condensing set-valued map with nonempty closed acyclic values. Then $F$ has a fixed point.

\section{Coincidence point}

Now we present a coincidence point theorem for condensing set-valued self-maps.

Theorem 2.1. Let $M$ be a hyperconvex metric space and $F: M \multimap M$ be an upper semicontinuous condensing set-valued map with nonempty closed acyclic values. Let $G: M \multimap M$ be an onto, quasiadmissible set-valued map for which $G(A)$ is closed for each closed set $A \subseteq M$. Assume that $G^{-}: M \multimap M$ is a 1-set contraction. Then there exists an $x_{0} \in M$ with

$$
F\left(x_{0}\right) \cap G\left(x_{0}\right) \neq \varnothing .
$$

Proof. Since

$$
F\left(x_{0}\right) \cap G\left(x_{0}\right) \neq \varnothing \Longleftrightarrow x_{0} \in G^{-}\left(F\left(x_{0}\right)\right)=\left\{x \in M: G(x) \cap F\left(x_{0}\right) \neq \varnothing\right\},
$$


then the conclusion follows if we show that the set-valued map $H(x)=G^{-}(F(x)): M \multimap$ $M$ has a fixed point. Since $G$ is onto, then $H(x) \neq \varnothing$. Since $F(x)$ is admissible and $G$ is quasiadmissible, then $H(x)$ is closed acyclic. Now we show that $H$ is upper semicontinuous. To show this, let $A$ be a closed subset of $M$. Then

$$
\begin{aligned}
H^{-}(A) & =\{x \in M: H(x) \cap A \neq \varnothing\} \\
& =\{x \in M:\{t \in M: G(t) \cap F(x) \neq \varnothing\} \cap A \neq \varnothing\} \\
& =\{x \in M: \exists a \in A \text { such that } G(a) \cap F(x) \neq \varnothing\} \\
& =\{x \in M: F(x) \cap G(A) \neq \varnothing\} \\
& =F^{-}(G(A)) .
\end{aligned}
$$

Since $F$ is upper semicontinuous and $G(A)$ is closed, then $H^{-}(A)=F^{-}(G(A))$ is closed. Hence $H$ is upper semicontinuous. Now we show that $H$ is condensing. To show this, let $A \subseteq M$ with $\alpha(A)>0$. Since $G^{-}$is 1 -set contraction and $F$ is condensing, then

$$
\alpha(H(A))=\alpha\left(G^{-}(F(A)) \leq \alpha(F(A))<\alpha(A)\right.
$$

Therefore, $H$ satisfies all conditions of Theorem 1.1 and so it has a fixed point.

Corollary 2.2. Let $M$ be a hyperconvex metric space and $f: M \rightarrow M$ be a continuous condensing map. Let $G: M \multimap M$ be an onto, quasiadmissible set-valued map for which $G(A)$ is closed for each closed set $A \subseteq M$. Assume that $G^{-}: M \multimap M$ is a 1-set contraction. Then there exists an $x_{0} \in M$ with

$$
f\left(x_{0}\right) \in G\left(x_{0}\right) .
$$

\section{Best approximation}

In this section, we extend some well-known best approximation theorems by involving a second set-valued map $G$.

Theorem 3.1. Let $M$ be a hyperconvex metric space and $X$ be a nonempty admissible subset of $M$. Let $F: X \multimap M$ be a Hausdorff continuous condensing set-valued map with nonempty bounded externally hyperconvex values and $G: X \multimap X$ be an onto, quasiadmissible set-valued map for which $G(A)$ is closed for each closed set $A \subseteq X$. Assume that $G^{-}: X \multimap X$ is a 1-set contraction. Then there exists an $x_{0} \in X$ such that

$$
d\left(G\left(x_{0}\right), F\left(x_{0}\right)\right)=\inf _{x \in X} d\left(x, F\left(x_{0}\right)\right) .
$$

Proof. Define a mapping $H: X \multimap M$ by

$$
H(x)=\bigcap_{\epsilon>\epsilon(x)}\left(N_{\epsilon}(X) \cap F(x)\right),
$$


where $\epsilon(x)=\inf \left\{\epsilon>0: N_{\epsilon}(X) \cap F(x) \neq \varnothing\right\}$. The values of $H$ are nonempty and externally hyperconvex [13, page 408, Theorem 5.4]. From [8, Lemma 1],

$$
D\left(N_{\epsilon(x)}(X) \cap F(x), N_{\epsilon(y)}(X) \cap F(y)\right) \leq D(F(x), F(y)) .
$$

Hence $D(H(x), H(y)) \leq D(F(x), F(y))$. Since $F$ is Hausdorff continuous, this implies that $H$ is also continuous in the Hausdorff metric. By a selection result in [16, Theorem 1], there is a mapping $h: X \rightarrow M$ such that $h(x) \in H(x)$ for each $x \in X$ and $d(h(x), h(y)) \leq$ $D(H(x), H(y))$ for each $x, y \in X$. Note $h$ is continuous. Since $h(x) \in H(x) \subseteq F(x), h$ is also condensing. The admissible set $X$ is a proximinal nonexpansive retract of $M$ [14] and we denote the retraction by $P_{X}: M \rightarrow X$. It follows that the mapping $P_{X}(h(\cdot)): X \rightarrow X$ is continuous and condensing, and therefore, by Corollary 2.2, there exists an $x_{0} \in X$ such that $P_{X}\left(h\left(x_{0}\right)\right) \in G\left(x_{0}\right)$. Fix $x \in X$. Now we show that $\epsilon(x)=d(X, F(x))$. Let $\epsilon>\epsilon(x)$ and let $y_{\epsilon} \in N_{\epsilon}(X) \cap F(x)$. Then $d(X, F(x)) \leq d\left(X, y_{\epsilon}\right) \leq \epsilon$. We can do this argument for each $\epsilon>\epsilon(x)$ so, therefore, $d(X, F(x)) \leq \epsilon(x)$. Suppose now that $d(X, F(x))<\epsilon(x)$. Then there exists a $y \in F(x)$ such that $d(X, F(x)) \leq d(X, y) \equiv \epsilon<\epsilon(x)$. Thus $y \in N_{\epsilon}(X) \cap F(x) \neq \varnothing$. This is a contradiction. Fix $n \in\{1,2, \ldots\}$ and let $\epsilon_{n}=d\left(X, F\left(x_{0}\right)\right)+1 / n$; note $\epsilon_{n}>\epsilon\left(x_{0}\right)$. Then since $h\left(x_{0}\right) \in H\left(x_{0}\right)$, we have $h\left(x_{0}\right) \subseteq N_{\epsilon_{n}}(X)$ so $d\left(X, h\left(x_{0}\right)\right) \leq \epsilon_{n}=d\left(X, F\left(x_{0}\right)\right)+1 / n$. We can do this for each $n$ so

$$
d\left(X, h\left(x_{0}\right)\right) \leq d\left(X, F\left(x_{0}\right)\right)
$$

Since $h\left(x_{0}\right) \in F\left(x_{0}\right)$ we get

$$
d\left(X, h\left(x_{0}\right)\right)=d\left(X, F\left(x_{0}\right)\right)
$$

Therefore, we have since $\left.P_{\mathbf{X}}\left(h\left(x_{0}\right)\right) \in G\left(x_{0}\right)\right)$ and $h\left(x_{0}\right) \in F\left(x_{0}\right)$ that

$$
\begin{aligned}
d\left(G\left(x_{0}\right), F\left(x_{0}\right)\right) & \leq d\left(P_{X}\left(h\left(x_{0}\right)\right), F\left(x_{0}\right)\right) \\
& \leq d\left(P_{X}\left(h\left(x_{0}\right)\right), h\left(x_{0}\right)\right) \\
& =d\left(X, h\left(x_{0}\right)\right),
\end{aligned}
$$

since $X$ is a proximity retract of $M$. Thus

$$
d\left(G\left(x_{0}\right), F\left(x_{0}\right)\right) \leq d\left(X, h\left(x_{0}\right)\right)=d\left(X, F\left(x_{0}\right)\right) .
$$

Since $G\left(x_{0}\right) \subseteq X$ then

$$
d\left(G\left(x_{0}\right), F\left(x_{0}\right)\right)=\inf _{x \in X} d\left(x, F\left(x_{0}\right)\right) .
$$

Remark 3.2. Let $X$ be a nonempty compact admissible subset of a hyperconvex metric space $M$ and let $G: X \rightarrow X$ be an isometry. We show that $G$ satisfies all the conditions of 
Theorem 3.1. Since $X$ is compact and $G: X \rightarrow X$ is an isometry, then $G$ is onto. Now we show that $G$ is quasiadmissible. Let $A$ be an admissible subset of $X$. Since $G$ is an isometry, then $G^{-}(A)=G^{-1}(A)$ is admissible and so is closed and acyclic. Let $A \subseteq X$ be closed, then $A$ is compact. Since $G$ is continuous, then $G(A)$ is compact and so is closed. Since $X$ is compact, then $G^{-1}: X \multimap X$ is a 1-set contraction (note for each $A \subseteq X, \alpha\left(G^{-1}(A)\right)=\alpha(A)=0$ ).

If we take $G=I$, then Theorem 3.1 reduces to the following result of Markin and Shahzad [2].

Corollary 3.3. Let $M$ be a hyperconvex metric space and $X$ be a nonempty admissible subset of $M$ and $F: X \multimap M$ be a Hausdorff continuous condensing set-valued map with nonempty bounded externally hyperconvex values. Then there exists an $x_{0} \in X$ such that

$$
d\left(x_{0}, F\left(x_{0}\right)\right)=\inf _{x \in X} d\left(x, F\left(x_{0}\right)\right)
$$

Proof. It suffices to show that $G=I$ satisfies the conditions of Theorem 3.1. The identity mapping $I: M \rightarrow M$ is onto and $I(A)=A$ is closed for each closed set $A \subseteq M$. Let $A$ be an admissible subset of $M$. Then $I^{-}(A)=A$ is admissible and so is acyclic [15, Lemma 5.2]. Thus $I$ is a quasiadmissible map. Finally, since $\alpha\left(I^{-}(A)\right)=\alpha(A)$ for each subset $A$ of $M$, then $I^{-}: M \rightarrow M$ is a 1-set contraction map.

The following is a coincidence point theorem for condensing nonself-set-valued maps.

Corollary 3.4. Let $M$ be a hyperconvex metric space and $X$ be a nonempty admissible subset of $M$. Assume the mappings F, G are compact valued and satisfy the conditions of Theorem 3.1. Assume that $F(x) \cap X \neq \varnothing$ for $x \in X$. Then there exists an $x_{0} \in X$ such that

$$
F\left(x_{0}\right) \cap G\left(x_{0}\right) \neq \varnothing .
$$

Proof. By Theorem 3.1, there exists an $x_{0} \in X$ with $d\left(G\left(x_{0}\right), F\left(x_{0}\right)\right)=\inf _{x \in X} d\left(x, F\left(x_{0}\right)\right)$. Since $F\left(x_{0}\right) \cap X \neq \varnothing$, then $\inf _{x \in X} d\left(x, F\left(x_{0}\right)\right)=0$. Thus $d\left(G\left(x_{0}\right), F\left(x_{0}\right)\right)=0$. Therefore, $F\left(x_{0}\right) \cap G\left(x_{0}\right) \neq \varnothing$.

\section{Best proximity pairs}

In this section, we obtain a best proximity pair theorem for condensing set-valued maps in hyperconvex metric spaces.

Theorem 4.1. Let $M$ be a hyperconvex metric space, $A$ be an admissible subset, and $B$ be a bounded externally hyperconvex subset of $M$. Let $G: A_{0} \multimap A_{0}$ an onto, quasiadmissible set-valued map for which $G(C)$ is closed for each closed set $C \subseteq A_{0}$. Assume that $G^{-}: A_{0} \multimap A_{0}$ is a 1-set contraction. Assume the mapping $F: A \multimap B$ is condensing, Hausdorff continuous with nonempty admissible values. Assume that $F(x) \cap B_{0} \neq \varnothing$ for each $x \in A_{0}$. Then there exists an $x_{0} \in A_{0}$ such that

$$
d\left(G\left(x_{0}\right), F\left(x_{0}\right)\right)=d(A, B) .
$$

Proof. By [2, Lemma 5.1], $A_{0}$ and $B_{0}$ are externally hyperconvex and nonempty. Define a mapping $H: A_{0} \multimap B_{0}$ by $H(x)=F(x) \cap B_{0}$. Since $A_{0}=\bigcap_{n=1}^{\infty} N_{d(A, B)+1 / n}(B) \cap A=A \cap N_{d(A, B)}(B)$ 
and $B_{0}=\bigcap_{n=1}^{\infty} N_{d(A, B)+1 / n}(A) \cap B=B \cap N_{d(A, B)}(A)$ [2, Lemma 5.1], then by [9, Lemma 1], we have $D\left(F(x) \cap B_{0}, F(y) \cap B_{0}\right) \leq D(F(x), F(y))$. Since $F$ is Hausdorff continuous, this implies that $H$ is continuous in the Hausdorff metric. Since $H(x)$ is externally hyperconvex for each $x \in A_{0}$, by a selection result in [16], there is a continuous mapping $h: A_{0} \rightarrow B_{0}$ such that $h(x) \in H(x)$ for each $x \in A_{0}$. Since $h(x) \in F(x), h$ is also condensing. The admissible set $A$ is a proximinal nonexpansive retract of $M$ and we denote the retraction by $P_{A}: M \rightarrow A$. Note $P_{A}\left(B_{0}\right) \subseteq A_{0}$. To see this note, if $y \in B_{0}$, then there is an $x \in A$ such that $d(x, y)=d(A, B)$. Thus $d\left(y, P_{A}(y)\right)=d(y, A) \leq d(y, x)=d(A, B)$ so we have $d\left(y, P_{A}(y)\right)=d(A, B)$ and so $P_{A}(y) \in A_{0}$. Since externally hyperconvex subset of $M$ is hyperconvex [13, page 398, Theorem 3.10], then $A_{0}$ is a hyperconvex metric space. Now the mapping $P_{A}(h(\cdot)): A_{0} \rightarrow A_{0}$ is continuous and condensing, and therefore, by Corollary 2.2, there exists an $x_{0} \in A$ such that $P_{A}\left(h\left(x_{0}\right)\right) \in G\left(x_{0}\right)$. Therefore, since $P_{A}\left(h\left(x_{0}\right)\right) \in A_{0}$, we have $d\left(P_{A}\left(h\left(x_{0}\right)\right), h\left(x_{0}\right)\right) \leq d\left(x, h\left(x_{0}\right)\right)$, for each $x \in A_{0}$. Since $h\left(x_{0}\right) \in B_{0}$, there is an $a_{0} \in A$ such that $d\left(a_{0}, h\left(x_{0}\right)\right)=d(A, B)$, and therefore, $B\left(h\left(x_{0}\right), d(A, B)\right) \neq \varnothing$. Furthermore, since $A_{0}=A \cap N_{d(A, B)}(B)$, then it follows from the external hyperconvexity of $N_{d(A, B)}(B)$ that $\left(B\left(h\left(x_{0}\right), d(A, B)\right) \cap A\right) \cap N_{d(A, B)}(B) \neq \varnothing$ (note $B\left(h\left(x_{0}\right), d(A, B)\right) \cap A$ is admissible) [16, Lemma 2]. Let $a_{1} \in B\left(h\left(x_{0}\right), d(A, B)\right) \cap A \cap N_{d(A, B)}(B)$. Then $a_{1} \in A$ and $d\left(a_{1}, h\left(x_{0}\right)\right)=d(A, B)$. Since $h\left(x_{0}\right) \in B_{0} \subseteq B$, then we have $a_{1} \in A_{0}$. Therefore, from the above, we have

$$
d\left(P_{A}\left(h\left(x_{0}\right)\right), h\left(x_{0}\right)\right) \leq d\left(a_{1}, h\left(x_{0}\right)\right)=d(A, B) .
$$

However, note also since $G\left(x_{0}\right) \subseteq A, F\left(x_{0}\right) \subseteq B, P_{A}\left(h\left(x_{0}\right)\right) \in G\left(x_{0}\right)$ and $h\left(x_{0}\right) \in F\left(x_{0}\right)$ that

$$
\begin{aligned}
d(A, B) & \leq d\left(G\left(x_{0}\right), F\left(x_{0}\right)\right) \\
& \leq d\left(P_{A}\left(h\left(x_{0}\right)\right), h\left(x_{0}\right)\right) \\
& \leq d\left(a_{1}, h\left(x_{0}\right)\right) \\
& =d(A, B) .
\end{aligned}
$$

Thus

$$
d\left(G\left(x_{0}\right), F\left(x_{0}\right)\right)=d(A, B)
$$

As a special case of Theorem 4.1, we obtain the following result of Markin and Shahzad [2].

Theorem 4.2. Let $M$ be a hyperconvex metric space, $A$ be an admissible subset, and $B$ be a bounded externally hyperconvex subset of $M$. Assume the mapping $F: A \multimap B$ is condensing, Hausdorff continuous with nonempty admissible values. Assume that $F(x) \cap B_{0} \neq \varnothing$ for each $x \in A_{0}$. Then there exists an $x_{0} \in A_{0}$ such that

$$
d\left(x_{0}, F\left(x_{0}\right)\right)=d(A, B) .
$$

\section{References}

[1] M. A. Khamsi, "KKM and Ky Fan theorems in hyperconvex metric spaces," Journal of Mathematical Analysis and Applications, vol. 204, no. 1, pp. 298-306, 1996. 
[2] J. T. Markin and N. Shahzad, "Best approximation theorems for nonexpansive and condensing mappings in hyperconvex metric spaces," Nonlinear Analysis: Theory, Methods \& Applications, vol. 70, no. 6, pp. 2435-2441, 2009.

[3] Z. D. Mitrović, "On scalar equilibrium problem in generalized convex spaces," Journal of Mathematical Analysis and Applications, vol. 330, no. 1, pp. 451-461, 2007.

[4] S. Park, "Fixed point theorems in hyperconvex metric spaces," Nonlinear Analysis: Theory, Methods $\mathcal{E}$ Applications, vol. 37, no. 4, pp. 467-472, 1999.

[5] A. Amini-Harandi and A. P. Farajzadeh, "A best approximation theorem in hyperconvex metric spaces," Communications on Applied Nonlinear Analysis, vol. 70, no. 6, pp. 2453-2456, 2009.

[6] A. Amini-Harandi, A. P. Farajzadeh, D. O'Regan, and R. P. Agarwal, "Fixed point theory for $\alpha$ condensing set valued maps in hyperconvex metric spaces," Communications on Applied Nonlinear Analysis, vol. 15, no. 2, pp. 39-46, 2008.

[7] W. A. Kirk, B. Sims, and G. X.-Z. Yuan, "The Knaster-Kuratowski and Mazurkiewicz theory in hyperconvex metric spaces and some of its applications," Nonlinear Analysis: Theory, Methods $\mathcal{E}$ Applications, vol. 39, no. 5, pp. 611-627, 2000.

[8] J. T. Markin, "A best approximation theorem for nonexpansive set-valued mappings in hyperconvex metric spaces," The Rocky Mountain Journal of Mathematics, vol. 35, no. 6, pp. 2059-2063, 2005.

[9] J. T. Markin, "Best approximation and fixed point theorems in hyperconvex metric spaces," Nonlinear Analysis: Theory, Methods \& Applications , vol. 63, no. 5-7, pp. 1841-1846, 2005.

[10] W. A. Kirk, S. Reich, and P. Veeramani, "Proximinal retracts and best proximity pair theorems," Numerical Functional Analysis and Optimization, vol. 24, no. 7-8, pp. 851-862, 2003.

[11] N. Aronszajn and P. Panitchpakdi, "Extension of uniformly continuous transformations and hyperconvex metric spaces," Pacific Journal of Mathematics, vol. 6, pp. 405-439, 1956.

[12] R. Sine, "Hyperconvexity and approximate fixed points," Nonlinear Analysis: Theory, Methods $\mathcal{E}$ Applications, vol. 13, no. 7, pp. 863-869, 1989.

[13] W. A. Kirk and B. Sim, Eds., Handbook of Metric Fixed Point Theory, Kluwer Academic Publishers, Dordrecht, The Netherlands, 2001.

[14] R. Sine, "Hyperconvexity and nonexpansive multifunctions," Transactions of the American Mathematical Society, vol. 315, no. 2, pp. 755-767, 1989.

[15] J.-H. Kim and S. Park, "Comments on some fixed point theorems in hyperconvex metric spaces," Journal of Mathematical Analysis and Applications, vol. 291, no. 1, pp. 154-164, 2004.

[16] M. A. Khamsi, W. A. Kirk, and C. M. Yañez, "Fixed point and selection theorems in hyperconvex spaces," Proceedings of the American Mathematical Society, vol. 128, no. 11, pp. 3275-3283, 2000. 